\title{
Evanescent-wave dynamic light scattering at an oil-water interface: Diffusion of interface-adsorbed colloids
}

\author{
Antonio Stocco, ${ }^{1}$ Tahereh Mokhtari, ${ }^{2}$ Günter Haseloff, ${ }^{1}$ Andreas Erbe, ${ }^{1, *}$ and Reinhard Sigel ${ }^{2, \dagger}$ \\ ${ }^{1}$ Max Planck Institute of Colloids and Interfaces, D-14476 Golm, Germany \\ ${ }^{2}$ Adolphe Merkle Institute, $\mathrm{CH}-1700$ Fribourg, Switzerland
}

\begin{abstract}
A light-scattering goniometer for evanescent-wave dynamic light scattering (EWDLS) measurements at a liquid-fluid interface is introduced, and used for measurements on two charge-stabilized polystyrene colloid systems adsorbed to alkane-water interfaces. The goniometer allows an independent variation of the penetration depth and the scattering vector components parallel and perpendicular to a liquid-fluid interface. The possible illumination geometries are compared. Ellipsometry at the liquid-fluid interface is implemented as a complementary tool. In EWDLS measurements, the absence of diffusive motion perpendicular to the interface is demonstrated, which confirms the adsorption of the particles. The two-step decay of the autocorrelation function is interpreted in terms of diffusion within a two-dimensional interface lattice of colloidal particles, stabilized by repulsive electrostatic interactions, and a desorption process. A significant slowing down of the in-plane diffusion of the colloids as compared to the bulk diffusion is observed.
\end{abstract}

\section{INTRODUCTION}

Dynamic light scattering (DLS) is a widely applied technique for the investigation of bulk dynamics of liquid samples [1,2]. It combines a huge experimental window in the time domain covering many decades with an excellent sensitivity, owing to the high scattering contrast present at optical wavelengths. Although the spatial resolution is limited, it is often possible to scale up the scattering particles and bring the length scale of interest into the experimental window $[3,4]$.

DLS measurements at a solid-liquid interface with a similar spatial and temporal resolution and the same high contrast were introduced by Lan, Ostrowsky, and Sornette more than 20 years ago [5]. An evanescent wave created in total internal reflection geometry illuminates only a slice at the interface within the medium of lower refractive index. The technique is often addressed as evanescent-wave dynamic light scattering (EWDLS) [6]. The thickness of the slice is comparable to the light wavelength and therefore to the experimental resolution parallel to the interface. There have been a number of investigations on colloid diffusion close to a solid or decorated wall [7-9], on polymer brush dynamics [10], and on liquid-crystal interfaces $[11,12]$. EWDLS measurements on the diffusion of "furry disks" formed by diblock copolymers on top of a water-air interface were reported by Lin, Rice, and Weitz [13]. A recent review provides an overview on further EWDLS investigations [14].

In this paper, EWDLS measurements on the liquid-liquid interface are presented. Instead of a solid glass substrate, the high refractive medium is formed by a liquid. This approach is an extension to previous EWDLS measurements, because it targets another type of interface. Other phenomena can be

\footnotetext{
${ }^{*}$ Present address: Max-Planck-Institut für Eisenforschung, D-40237 Düsseldorf, Germany.

${ }^{\dagger}$ Author to whom correspondence should be addressed. reinhard.sigel@unifr.ch
}

studied, for example, an exchange of particles through the interface or interactions of particles with capillary waves. In the present work, the two-dimensional (2D) diffusion of colloidal particles adsorbed to the interface is investigated. The first system under investigation was composed of polystyrene (PS) colloids of hydrodynamic radius $R=52.3 \mathrm{~nm}$ at a water-dodecane interface. These colloids are electrostatically stabilized by sulfate groups. For improved purity of the oil phase and larger scattering signal, we subsequently switched to a water-heptane interface and PS particles of larger radius $R=85 \mathrm{~nm}$, where the electrostatic stabilization is based on adsorbed perfluorooctanoate (PFO) groups. A detailed investigation of the interface adsorption mechanisms of these two systems by ellipsometry will be presented separately.

In bulk measurements, the investigation of diffusion in the dilute regime is the main application of DLS. The resulting diffusion coefficient $D$ is connected via the Stokes-Einstein relation to the solvent viscosity and the hydrodynamic radius of the particle [1]. For particles pinned to a liquid-fluid interface, the in-plane diffusion constant $D_{\|}$yields information about interfacial friction and particle mobility. These parameters are important for interfacial relaxation processes, and relevant for a number of phenomena, such as foam stability.

To date, light-scattering measurements at the liquid-air interface mainly target the investigation of capillary waves [15]. In this case, a contribution is expected only at small scattering angles and, for total internal reflection geometry, at the critical angle. Usually, sophisticated small-angle light-scattering goniometers are used for such experiments. The investigation of the in-plane particle diffusion described here is an experimental counterpart in the wide angle range. The contribution of capillary waves of the wavelength selected by the scattering vector is negligible in this angular range. For a distinction of particle diffusion parallel and perpendicular to the interface, an independent variation of the scattering vector components parallel and perpendicular to the interface is indispensable. The scattering instrument presented in 
this work achieves this task differently than an experiment described previously by Holmqvist, Dhont, and Lang [8]

The paper is organized as follows: Section II briefly repeats EWDLS and compares the two possible observation geometries. The new apparatus is presented in Sec. III. After a description of experimental details in Sec. IV, an investigation of the particle diffusion in the interface by EWDLS is described and discussed in Sec. V. Details of the experimental coordinates and the alignment procedure are presented in Appendixes A and B, respectively.

\section{COMPARISON OF SCATTERING GEOMETRIES}

EWDLS is based on a total internal reflection geometry. The illuminating light beam enters through medium 1 with the higher refractive index $n_{1}$ and hits the interface to medium 2 with lower refractive index $n_{2}$. The refraction is described by Snell's law,

$$
\frac{\sin \varphi}{\sin \varphi^{\prime}}=\frac{n_{2}}{n_{1}}
$$

where $\varphi$ and $\varphi^{\prime}$ are the angles of the incident and the refracted beam to the interface normal. For a perpendicular refraction $\varphi^{\prime}=90^{\circ}, \varphi$ reaches the critical angle $\varphi_{0}$ of total internal reflection. The normal component $k_{i \perp}^{\prime}$ of the wave vector in medium 2 reads

$$
k_{i \perp}^{\prime}=n_{2} k_{0} \cos \left(\varphi^{\prime}\right)=n_{1} k_{0} \sqrt{\sin ^{2} \varphi_{0}-\sin ^{2} \varphi},
$$

where $k_{0}=2 \pi \lambda^{-1}$ is the vacuum wave vector and $\lambda$ is the light wavelength. For $\varphi<\varphi_{0}$, there is a plane wave with an oscillatory behavior $\exp \left(i k_{i \perp}^{\prime} z\right)$. For $\varphi>\varphi_{0}$, Eq. (2) has a purely imaginary result and the exponential decay $\exp (-p z)$ of the evanescent wave results, where $p=\left|k_{i \perp}^{\prime}\right|$ indicates the inverse penetration depth. In this case, $\varphi^{\prime}$ calculated from Eq. (1) becomes a complex number and no longer has a geometric meaning.

For a proper understanding of the EWDLS experiment, the detection optics is important, and it affects the scattering geometry. There are two possibilities for the detection of the scattered light. In most investigations, a detection through the low refractive-index medium 2 is selected [7-10], while only for a few contributions a measurement through the high refractive-index medium 1 is chosen [12]. The two geometries are compared in Fig. 1, where the scattering of a particle at a depth $d$ in medium 2 and the bulk scattering of medium 1 are considered. For the detection through medium 1 at a detector angle $\psi$ as shown in Fig. 1(a), the interface leads to refraction of the light scattered by the sphere in medium 2. Because of momentum conservation, the wave-vector component parallel to the interface remains unchanged [16], and only the effect on the normal component has to be considered for correct assignment of the scattering vector. For static light scattering, an intensity transmission coefficient has to be considered. For the scattering background of medium 1, there are two wave vectors $\mathbf{k}_{i, 1}, \mathbf{k}_{i, 2}$ for the incident light and two wave vectors $\mathbf{k}_{s, 1}, \mathbf{k}_{s, 2}$ of scattered light because of reflections at the interface. The intensity results as the interference signal of all four scattering vectors $\mathbf{q}_{m, n}=\mathbf{k}_{i, m}-\mathbf{k}_{s, n}(m, n \in$ $\{1,2\})$, calculated as the difference of one incident and one
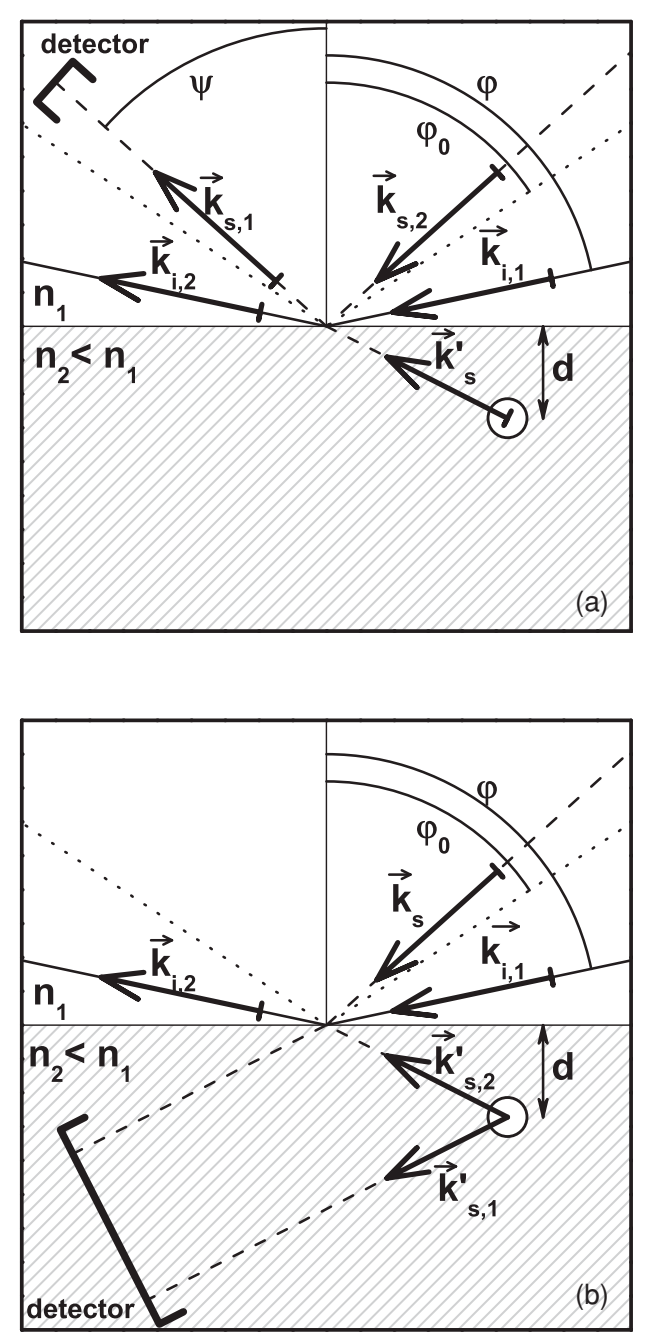

FIG. 1. Comparison of EWDLS geometries. In (a), the scattered light is detected through the medium of higher refractive index $n_{1}$; in (b) the scattering is observed through the phase of lower refractive index $n_{2}$. Details are described in the text.

scattered wave vector, respectively. Besides the interference, the reflection of incident and scattered light additionally increases the average scattering background.

Concerning the background scattering of medium 1 , the situation simplifies with detection through medium 2, as shown in Fig. 1(b). There are two relevant wave vectors $\mathbf{k}_{i, 1}, \mathbf{k}_{i, 2}$ for the incident light, while only scattered light of a single wave vector $\mathbf{k}_{s}$ reaches the detector. Thus, there is an interference between the scattering of only two different scattering vectors. For light scattered by the particle in medium 2, however, there is now interference of the scattering directly to the detector (wave vector $\mathbf{k}_{s, 1}^{\prime}$ ) and a second contribution, which is scattered toward the interface (wave vector $\mathbf{k}_{s, 2}^{\prime}$ ), where it is reflected to the detector. The signal of a scattering particle at a depth $d$ is no longer determined solely by the exponential intensity profile of the evanescent wave, but a modification of the interference has to be taken into account. As an illustration, the scattering of a small spherical particle located at a depth $d$ in the normal direction is considered. The two scattering contributions have the same amplitude because the two involved scattering vectors are mirror images, and the scattered intensity at the detector 
position $z_{D}$ is proportional to the coefficient

$$
F=e^{-2 p d}\left|\frac{e^{i k_{f \perp}^{\prime}\left(z_{D}-d\right)}}{z_{D}-d}+r^{\prime} \frac{e^{i k_{f \perp}^{\prime}\left(z_{D}+d\right)}}{z_{D}+d}\right|^{2} .
$$

The first factor describes the depth dependence owing to the evanescent wave. Additionally, there is the oscillatory interference term in the second factor, which contains the normal component of the wave vector of the scattered light $k_{s \perp}^{\prime}$. The amplitude reflection coefficient $r^{\prime}=\left(n_{2}-n_{1}\right) /\left(n_{2}+n_{1}\right)$ is negative [16], so there is partially destructive interference for a particle close to the interface and constructive interference for a particle a bit further outside. The interface sensitivity of EWDLS is lowered for an observation through medium 2. The effect causes a significant deviation from the exponential depth dependence of the evanescent wave alone, because it is first order in $r^{\prime}$. The common choice of a large difference $n_{2}-n_{1}$ for good depth resolution increases $r^{\prime}$ also. A variation of the detection angle affects the depth resolution through $r^{\prime}$, even though the penetration depth of the evanescent wave is unchanged.

Figure 1 illustrates the case where all vectors $\mathbf{k}_{i, m}, \mathbf{k}_{s, m}$, $\mathbf{k}_{s, m}^{\prime}, \mathbf{q}_{m, n}(m, n \in\{1,2\})$ and the interface normal $\hat{n}$ are in a single plane. The discussed interference of contributions with different scattering vector owing to the reflection at the interface also occurs in the general case, where the planes of incidence and the planes of reflection no longer coincide. These planes are spanned by $\hat{n}$ and an incident or a scattered wave vector, respectively. The general case is more involved owing to the polarization dependence of the scattering amplitudes. Further, the apparently large detector size in Fig. 1(b) originates only from the schematic representation. In a real experiment, the detector is much larger than $d$ and it is located in the far field.

The apparatus presented in the next paragraph allows the detection of the scattered light through either medium. We prefer the detection through the high refractive-index medium 1 .

There are experimental benefits of the liquid-liquid interface or the liquid-air interface (the two cases are summarized as the liquid-fluid interface) compared to the solid-liquid interface, where measurements are quite challenging. It is probably because of the experimental difficulties that the number of EWDLS investigations at the solid-liquid interface is remarkably small compared to the wide application of DLS bulk measurements, which fills entire textbooks. For investigations of a solid-liquid interface, a solid substrate is inevitably present in the scattering volume. In previous experiments, glass has been used exclusively for that purpose. The glass's interfacial roughness and bulk scattering contributions both cause a scattering background, which is superimposed to the signal of interest originating from interfacial dynamics. Although the glass scattering cross section is usually much smaller than the sample scattering cross section, the contribution from the substrate is often dominant, even for an ideally flat interface. The significant glass contribution is owing to the unfavorable volume ratio within the scattering volume. While the evanescent wave intentionally restricts scattering volume on the sample side of the interface, the width of the scattering volume within the glass is determined by the much larger beam diameter. The illuminated glass volume is typically two orders of magnitude larger than the illuminated sample volume. In addition, the interface roughness in real experiments and the resulting scattering are often substantial. Because there is no dynamics in the glass substrate, its scattering consists of static laser speckles. Minute changes of the angles of incidence and detection result in a strong and erratic change in the scattering background. The unfavorable speckle pattern also complicates DLS measurements. It causes a mixing of the laser light to the dynamic signal of the sample and therefore acts as a local oscillator [1]. The fraction of local oscillator light affects the amplitude and the relaxation time of the intensity autocorrelation function of DLS measurements, where a change of up to a factor 2 might occur. A suitable data evaluation can remove this effect [8]; however, it relies on good data quality of the correlation function, where slow modes should be absent. With the static speckle background, a high mechanical and temperature stability of the experiment is required to avoid a slow mode. These problems are absent for the liquid-fluid interface. The scattering of the high refractive-index phase in this case has its own dynamics and therefore no longer consists of static laser speckles. There is no heterodyning of the DLS measurements and a smooth background contribution to static light scattering. Further, the interfacial tension between the liquids generates a flatness of the interface, which is far superior to any polished glass surface. The demands on mechanical stability and vibration damping, on the other hand, are comparable to the requirements for an investigation of the solid-liquid interface.

The scattering vector $\mathbf{q}$ is a more comprehensive quantity for EWDLS compared to bulk scattering measurements. For the latter, the magnitude $|\mathbf{q}|$ is the only relevant experimental parameter for measurements in isotropic media. In EWDLS, however, structural and dynamical sample properties in directions parallel and perpendicular to the interface are not necessarily the same. For a distinction, the parallel component $q_{\|}$and the real part of the normal component $q_{\perp}$ of $\mathbf{q}$ have to be considered separately. Further, the inverse penetration depth $p$ forms the imaginary part of $\mathbf{q}$. The occurrence of an imaginary contribution indicates that in the direction perpendicular to the interface the detection is not sharp in $\mathbf{q}$ space (the momentum space). The small dimension of the scattering volume in this direction is comparable to the light wavelength, which results in a strong smearing in $\mathbf{q}$ space.

\section{THREE-DIMENSIONAL SCATTERING GONIOMETER}

With $q_{\|}, q_{\perp}$, and $p$, there are three important experimental parameters. An independent variation of these parameters in an experiment is desirable. In a planar geometry with monochromatic illumination, a variation of the angles of incidence and detection opens up a 2D manifold in the threedimensional (3D) $\left(q_{\|}, q_{\perp}, p\right)$ space. The wavelength could be varied as a third parameter to access a $3 \mathrm{D}$ region of the $\left(q_{\|}, q_{\perp}, p\right)$ space. However, the variation is rather limited within the range of visible light. In addition, a wavelength dependence of a medium refractive index might complicate the situation. A 3D goniometer that allows the detection 


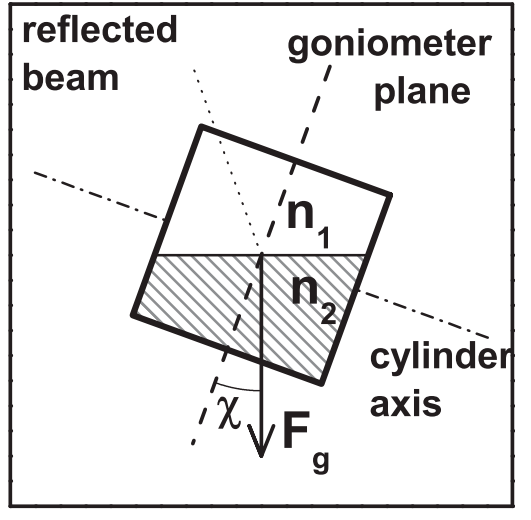

FIG. 2. Scheme (side view) of the experimental geometry. The lower half of a cylindrical cell contains a liquid with refractive index $n_{2}$, while the upper half contains air or another liquid of refractive index $n_{1}$. The earth gravitational force $\mathbf{F}_{g}$ keeps the interface in a horizontal orientation. The illumination of their interface and the detection of reflected, refracted, or scattered light relies on a planar two-armed goniometer, which operates in the goniometer plane. By tilting the whole setup by an angle $\chi$, scattered light outside the plane of reflection can be accessed.

of the scattering out of the plane of reflection overcomes these limitations. Such an apparatus accessing directly the polar coordinates in a spherical geometry for investigations of solid-liquid interfaces was realized by Holmqvist, Dhont, and Lang [8].

For the investigation of liquid-liquid or liquid-air interfaces we followed an alternate approach. The employed cylindrical geometry is shown in Fig. 2. The starting point is a planar twoarmed goniometer, where a laser arm carries the illumination light source and the detector arm allows the positioning of the detector. The two arms can be moved within the goniometer plane. A tilt of the whole goniometer out of a vertical orientation results in a geometry where the goniometer plane deviates from the plane of reflection. In this way, 3D out-of-plane scattering can be accessed. The approach relies on the liquid nature of the investigated samples. Gravity arranges a horizontal orientation of the liquid-fluid interface, independently of the tilt of the goniometer. The concept of a cylindrical geometry arose from accuracy considerations. While it is possible to manufacture a very perfect cylinder, it is more difficult to obtain a high-quality sphere. The accuracy of the geometric shape of the sample container limits the adjustment of an apparatus, and therefore a cylindrical geometry is of advantage. From a geometric point of view, the tilted cylindrical geometry is more involved than the direct technical implementation of cylindrical coordinates. Details of the required coordinate transformations are described in Appendix A. However, the cylindrical geometry has some advantages and turns out to be complementary to the apparatus built by Holmqvist, Dhont, and Lang [8]. A first advantage is an easier alignment, which potentially results in a better adjustment. It involves well-established procedures for the adjustment of bulk light-scattering setups, which are described in Appendix B. Further, because the illumination and the detection remain within the goniometer plane, all of the rest of a scattering cell can be covered. In this way a temperature control is feasible. In contrast, a true spherical geometry requires a larger open area, which reduces the efficiency of any thermostat. Finally, the realization of a tilted cylindrical geometry is less involved from a technical point of view compared to a 3D goniometer realizing spherical coordinates. The resulting apparatus is very light and therefore can be placed on an active vibration damping table TS140 (HWL, Ammerbuch, Germany). In comparison, the apparatus of Holmqvist, Dhont, and Lang has an impressive size and is much heavier. The damping is essential for a reliable investigation of highly mobile liquid-air interfaces.

Our apparatus is based on a two-armed goniometer "Multiskop" (Optrel, Kleinmachnow, Germany), which has a large central opening and allows convenient placement of samples [17]. The tilting is motor driven by a linear stage LF6 (isel, Eichenzell, Germany), where a leverage converts the translation to a tilt motion (see the inset of Fig. 3). A counterweight is fixed to the goniometer to keep the apparatus nearly in balance and to minimize the holding force of the linear stage. Mechanical adjustment tools (OWIS, Staufen, Germany) enable the adjustment of all six degrees of freedom for a centered port that carries the sample cell.

A schematic drawing of the optics is shown in Fig. 3. The goniometer arms are equipped with an optomechanical rail system (OWIS), which provides a high flexibility for experiments. The arms accommodate a folded beam geometry based on mirrors (four on the laser arm and two on the detector arm), where the optical paths pass the top side of the arms and return at the bottom sides. In this way, a maximum effective arm length of $\sim 90 \mathrm{~cm}$ from the center is realized on the arms of limited size. A long path length is important for ultrasmall-angle light-scattering experiments for the detection of capillary waves. The rail on top of the arm is fixed on a small translational stage (IKO Nippon Thompson, Tokyo, Japan). This stage allows a complete shift of preadjusted optics, e.g., to compensate for a changed refractive index of a sample owing to a temperature change. The optics is based on cylindrical lenses (focal length $f=150 \mathrm{~mm}$ ) in order to compensate the astigmatism introduced by the cylindrical scattering cell. A 25-mW HeNe laser (OWIS) with a wavelength $\lambda=633 \mathrm{~nm}$ is fixed parallel to the laser arm (Fig. 3(a) shows a perpedicular laser mounting which was used in the beginning). The goniometer arms include three motorized rotational stages (Physik Instrumente, Karlsruhe, Germany) that rotate a polarizer and a compensator on the laser arm and an analyzer on the detector arm. A combination of a fixed quarter-wave plate and a second quarter-wave plate rotating with the polarizer provides minimum intensity loss in the polarization optics [18]. The compensator is another quarter-wave plate necessary for ellipsometry [19], which yields complementary information to the scattering data. Ellipsometry at the liquid-liquid interface has been introduced only recently [20], based on submersed light guides. Here, this technique appears as a byproduct, where the sample cell provides advantages as an enclosed sample container and for temperature control. The polarizers are Glan-Thompson prisms (Bernhard Halle, Berlin, Germany) with an extinction ratio of $10^{-8}$ and less than 5 arc-seconds. beam deflection for minimized deviations upon rotation. While for ellipsometric measurements the compensator is set to an 

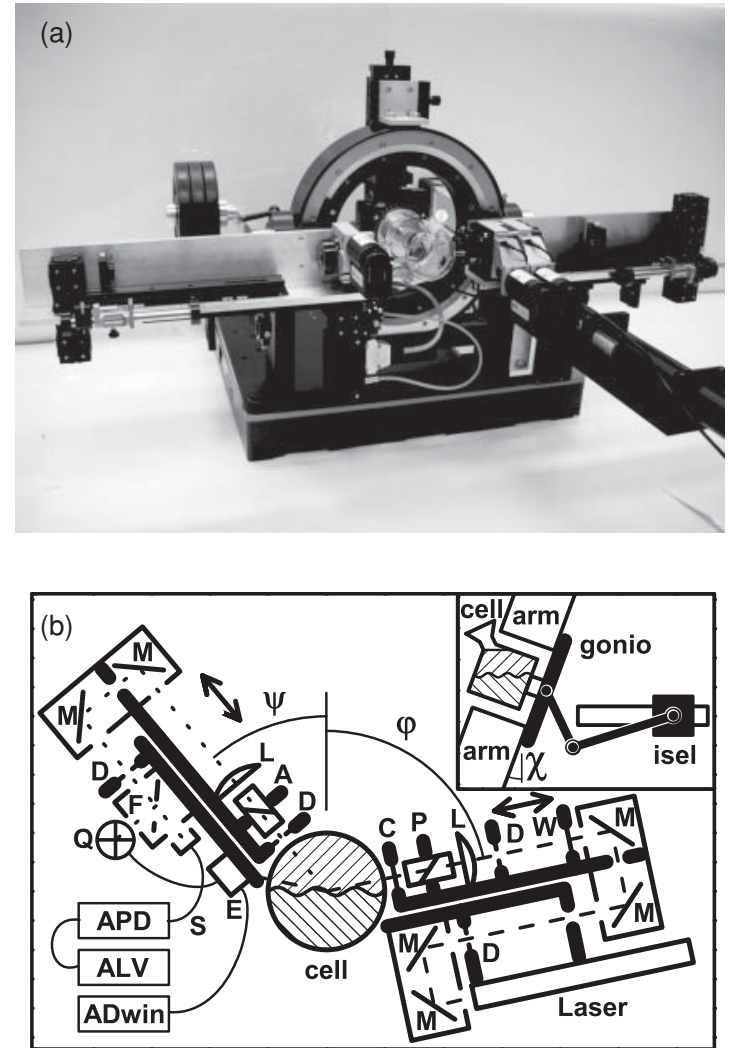

FIG. 3. Picture and sketch of the apparatus realizing the tilted plane geometry for 3D EWDLS. The laser beam (dashed line) on the laser arm (right-hand side, moved to the angle $\varphi$ ) and the detected light (dotted line) on the detection arm (left-hand side, deflected to the angle $\psi$ ) follow a path folded by mirrors (M) and defined by diaphragms (D). Weak focusing of the illumination light and collimation of the scattered or reflected light is implemented by cylindrical lenses (L), respectively. The option to adapt the distance of the optics on the top parts of both arms to the cell is indicated by arrows. A quarter-wave plate (W) creates circular polarization. The subsequent polarization optics consists of polarizer (P), compensator (C), and analyzer (A), driven by individual stepping motors. A motorized flipping mirror (F) allows computer-controlled switching of the detection between a quadrant diode $(\mathrm{Q})$ connected via a preamplifier (E) to the ADwin board or a single-mode fiber $(\mathrm{S})$ leading to an avalanche photodiode (APD) connected to a correlator (ALV). The inset shows a side view, where the leverage mechanism driven by a linear stage (isel) is outlined, which tilts the goniometer and the cylindrical cell by the angle $\chi$.

azimuthal angle of $45^{\circ}$ toward the plane of reflection, it is deactivated by parallel alignment with the polarizer in case of scattering measurements. Measurements at the direct beam (ellipsometry, reflection, transmission) employ a quadrant diode as detector. Here, the electronics of the Multiskop based on an ADwin board (Jäger, Lorsch, Germany) is adopted. The quadrant diode yields the intensity and the position of the detected beam. Scattering is detected by an avalanche photodiode (Perkin-Elmer). The application of a monomode fiber yields optimal contrast in DLS measurements [21], which are performed by using an ALV5000 correlator with fast extension (ALV, Langen, Germany). A motorized flipping mirror (OWIS) enables computer-controlled switching between the two detection modes.
The software for computer-controlled experiments was realized within Origin 7 (OriginLab Corporation, Northampton, MA), so measurements are directly connected to data evaluation and representation. The software allows batch processing of measurement tasks and gives direct access to polar coordinates and the $\left(q_{\|}, q_{\perp}, p\right)$ space. Additionally, helpful adjustment tools such as an automated search for the beam position based on a scan of a goniometer arm in connection with the quadrant diode signal are implemented. For high-accuracy nulling ellipsometry measurements, a 2D scan of the polarizer and analyzer, a fine calibration of the rotation-angle-specific transmission through the polarization optics, and a suitable fitting scheme was adopted from ellipsometric light scattering (ELS) [18]. The latter technique was developed for a characterization of thin layers on dispersed colloidal particles [22].

Sample cells were manufactured by the Max Planck Institute's glass blower workshop. For a low curvature of the interface in the center, cylindrical cells of $7.5 \mathrm{~cm}$ outer diameter and $7.5 \mathrm{~cm}$ total length are used (volume $V=270 \mathrm{~cm}^{3}$ ). These dimensions are recommended in the literature to minimize meniscus effects [15]. The cylindrical design leads to a limited change of interface area when the sample cell is tilted together with the goniometer. For a tilt up to $\chi=50^{\circ}$, the change in the interface area is only $13 \%$. For samples where this change is of significance, a spherical sample cell could be used. The cell is mounted via a ground glass fitting, where a metal counterpart (Vacom, Jena, Germany) forms the port in the goniometer. This clamping via a cone allows a reproducible insertion of the sample cell. A simple temperature system was realized by a heated hose (Isopad, Heidelberg, Germany).

The apparatus described has been employed for highresolution ellipsometry on bare liquid-fluid interfaces [23] and the detection of capillary-wave dynamics [24].

\section{EXPERIMENT}

\section{A. Material}

The persulfate stabilized PS latex "Harry" was prepared by emulsion polymerization in the presence of high peroxodisulfate concentration. After the synthesis, the latex was carefully ultrafiltrated to remove surfactant and residual initiator. Neither micelles nor free surfactant molecules were present in the latex suspension after ultrafiltration. Ionic end groups from the persulfate initiator ensure the colloidal stability of the particles. The "Harry" colloids have a hydrodynamic radius $R=52.3 \mathrm{~nm}$ and a zeta potential $\zeta=-50 \mathrm{mV}$. Synthesis and scattering characterization of the Eur62 colloids stabilized by adsorbed PFO groups with $R=85 \mathrm{~nm}$ are described in Ref. [18]. Measurements of $\zeta$ hint to a partial desorption of PFO for low colloid concentration $c$. Results vary from $\zeta=-65 \mathrm{mV}$ at $c=71 \mathrm{mg} / \mathrm{L}$ to $\zeta=-6.8 \mathrm{mV}$ at $c=0.05 \mathrm{mg} / \mathrm{L}$. Lower $c$ 's were not accessible with the used Malvern NanoZ Zetasizer with disposable polycarbonate cells (DTS1060). The phenomenological equation $\zeta^{-1}=\zeta_{0}^{-1}(1+$ $c_{0} / c$ ) with $\zeta_{0}=-60.2 \mathrm{mV}$ and $c_{0}=0.52 \mathrm{mg} / \mathrm{L}$ captures the trend.

\section{B. Sample preparation}

Dodecane-water interfaces covered by "Harry" colloids or heptane-water interfaces covered with Eur62 particles were 
prepared with filtered $(0.2 \mu \mathrm{m})$ double-distilled de-ionized water (milipore) and dodecane (Sigma-Aldrich, purity >99\%) or heptane (Acros Organics, 99\% + pure), respectively, as the oil phase. In the case of measurements on the "Harry" sample, a small amount of aqueous $\mathrm{NaN}_{3}$ solution $\left(10^{-7} \mathrm{wt} \%\right)$ was added to the water as a disinfecting agent to prevent the growth of microorganisms. The cell was half filled with water by using a syringe with a long needle. Then the oil was slowly added on top until the cell was completely filled. For a fine adjustment of the filling level, the cell was mounted to the apparatus with a laser and detector arm in reflection geometry $\left(\varphi=\psi=75^{\circ}\right.$; see Fig. 3). The height of the water-oil interface was adjusted by adding or removing water by using a syringe until the beam reflected at the interface hit the center of the quadrant diode sensor on the detector arm. Furthermore, the cleanliness of the interface was verified via static evanescent-wave light scattering within a range of $\psi=45^{\circ}-65^{\circ}$. In the presence of impurities, dust or bubbles at the interface, the photon count rate was significantly higher than the dark count rate. In such a case, the interface was cleaned with a long needle connected to a liquid pump (KNF Liquiport) via flexible tubing. The height of the interface was then readjusted by repeating the procedure mentioned above.

With a microsyringe, a volume of $V_{0}=50 \mu \mathrm{L}$ colloidal dispersion [initial concentration $c_{1}=250 \mathrm{mg} \mathrm{L}^{-1}$ ("Harry") or $c_{1}=440 \mathrm{mg} \mathrm{L}^{-1}$ (Eur62)] was gently added at different locations just below the interface ("Harry") or on the oil side directly above the interface (Eur62). For an improved particle distribution, the interface was carefully stirred (Eur62). The nominal colloid concentration $c=c_{1} V_{0} /(V / 2)$ in water resulted as $c=0.09 \mathrm{mg} \mathrm{L}^{-1}$ ("Harry") or $c=0.16 \mathrm{mg} \mathrm{L}^{-1}$ (Eur62). The system was left to equilibrate for $12 \mathrm{~h}$ ("Harry") or 2 days (Eur62) before the first measurements. The area fraction of the interface covered by the particles $\Phi=2.5 \%$ for "Harry" and $\Phi=1.0 \%$ for Eur62 was measured by ellipsometry. As compared to the total amount of added particles, these results indicate a complete adsorption of "Harry" and a partial adsorption for Eur62. A detailed discussion of the ellipsometry data analysis and the difference in adsorption mechanism for the two samples will be presented separately.

\section{RESULTS AND DISCUSSION}

For an investigation of the particle diffusion, the interface was illuminated through the high refractive oil phase with $\varphi>\varphi_{0}$, so the exponentially decaying profile of the evanescent wave builds up in the water phase. The scattered light was detected through the oil phase. For Eur62, the intensity autocorrelation functions $g_{2}(t)$ determined by the correlator had an intercept $\beta=\left[g_{2}(t \rightarrow 0)-1\right]$ above 0.95 , so no heterodyning is present. The transformation to field autocorrelation functions $g_{1}(t)$ was performed simply via the Siegert relation, as for bulk light scattering on ergodic samples [1]. For solid-liquid interfaces, in contrast, where immobile scattering centers in the solid phase or at the interface act as local oscillators that mix primary illumination to the scattered light, a more complex transformation is required [8]. The geometry with particles adsorbed to the interface is different from the case discussed in Sec. II, where the particle

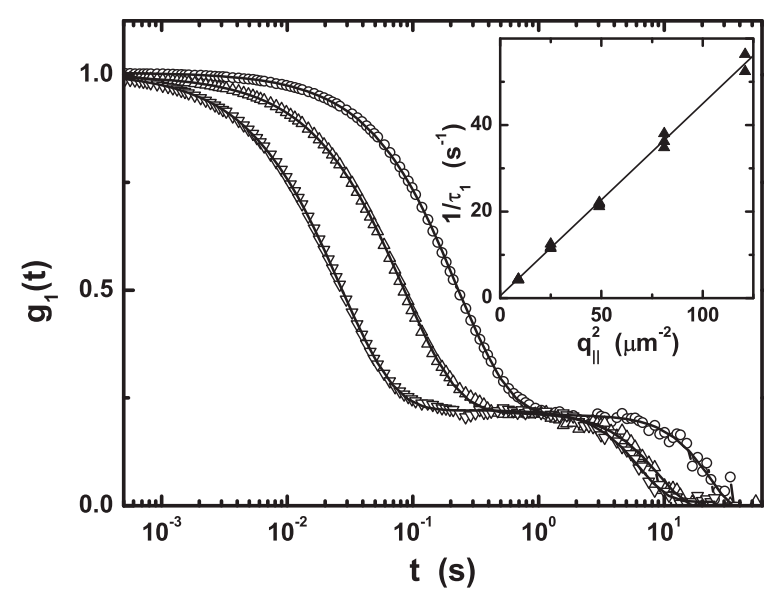

FIG. 4. Field correlation functions $g_{1}(t)$ and fits by two exponentials, respectively, for Eur62 colloids at the heptane-water interface under evanescent-wave illumination with penetration depth $p^{-1}=$ $2 \mu \mathrm{m}$. The perpendicular scattering vector component $q_{\perp}=2 \mu \mathrm{m}^{-1}$ is fixed, while the parallel scattering vector component $q_{\|}$varies ( $3 \mu \mathrm{m}^{-1}, \triangle 5 \mu \mathrm{m}^{-1}, \nabla 9 \mu \mathrm{m}^{-1}$ ). The inset shows the inverse relaxation time $\tau^{-1}$ of the fast mode for different $q_{\|}$with $p^{-1}$ and $q_{\perp}$ as before.

diffusion in the vicinity of a solid interface is considered. Still, the illumination by an evanescent wave is essential to achieve the sensitivity to interfacial phenomena. Because DLS performed in this work is based on the dynamics of fluctuations in scattering intensity, it does not rely on a model to calculate the scattering amplitudes of particles in the interface.

Figures 4 and 5 show $g_{1}(t)$ for Eur62 at the heptane-water interface. A slight rescaling was applied to adapt the intercept to 1. A two-step decay is observed with a fast relaxation that shifts with $q_{\|}$(Fig. 4) but is independent of $q_{\perp}$ (Fig. 5).

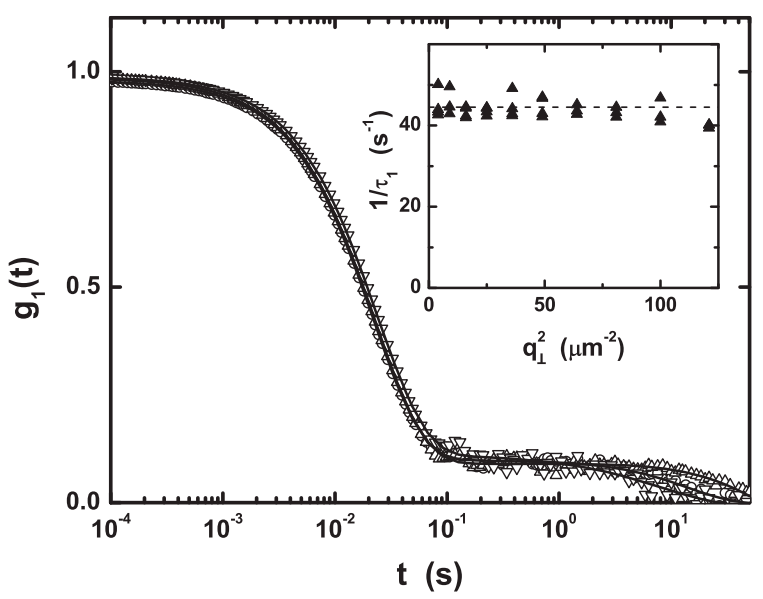

FIG. 5. Field correlation functions $g_{1}(t)$ and fits by two exponentials, respectively, for Eur62 colloids at the heptane-water interface under evanescent-wave illumination with penetration depth $p^{-1}=$ $2 \mu \mathrm{m}$. The parallel scattering vector component $q_{\|}=10 \mu \mathrm{m}^{-1}$ is fixed, while the perpendicular scattering vector component $q_{\perp}$ varies ( $\bigcirc \mu \mathrm{m}^{-1}, \triangle 5 \mu \mathrm{m}^{-1}, \nabla 10 \mu \mathrm{m}^{-1}$ ). The inset shows the inverse relaxation time $\tau^{-1}$ of the fast mode for different $q_{\perp}$ with $p^{-1}$ and $q_{\|}$ as before. 
For measurements at a clean, bare heptane-water interface, in contrast, $g_{1}(t)$ is flat and none of the two relaxation modes is present. For an analysis of $g_{1}(t)$ data, a sum of two exponentials provided a good description in a fitting procedure. The unchanged value of the inverse fast relaxation rate $\tau_{1}^{-1}$ for fixed values of $p^{-1}$ and $q_{\|}$but varying $q_{\perp}$ is shown in the inset of Fig. 5. This behavior demonstrates the 2D nature of the particle diffusion and thus the particle adsorption to the interface. The smearing in $q_{\perp}$ direction (see Sec. II) characterized by the inverse penetration depth $p=0.5 \mu \mathrm{m}^{-1}$ is much smaller than the width of the investigated $q_{\perp}$ range from 3 to $11 \mu \mathrm{m}^{-1}$, so the variation of $q_{\perp}$ is sufficiently wide to be conclusive. For a variation of $q_{\|}$, the inset of Fig. 4 displays the linear scaling of $\tau_{1}^{-1}$ with $q_{\|}^{2}$. The slope yields the in-plane diffusion constant $D_{\|}=(0.44 \pm 0.01) \mu \mathrm{m}^{2} / \mathrm{s}$. A small intercept $\tau_{0}^{-1}=(0.5 \pm 0.7) \mathrm{s}^{-1}$ is interpreted as the desorption rate of the particles from the interface. The functional form $\exp \left(-t / \tau_{0}\right) \exp \left(-q_{\|}^{2} D_{\|} t\right)$ of the fast mode resembles the superposition of translational and rotational diffusion observed in depolarized bulk light scattering. In this case, a decoupling approximation of the two movements leads to a diffusion contribution averaged over all particle orientations that scales with $\mathbf{q}^{2}$, and a relaxation rate of the particle orientation by rotational diffusion [1]. For our interface measurements, a similar interpretation assigns the slope $D_{\|}$ on the $q_{\|}^{2}$ scale to an averaged diffusion of adsorbed and desorbed particles, while a constant term $\tau_{0}$ is the relaxation rate of the exchange between adsorbed and desorbed state. Based on the bulk concentration, interface coverage, and particle radius given in Sec. IV B and the particle density $\rho_{P S}=1.05 \mathrm{~g} / \mathrm{cm}^{3}$ [25], the ratio of adsorbed to nonadsorbed particles in the scattering volume $A p^{-1}$ with a footprint area $A$ results as $\left[A \Phi /\left(\pi R^{2}\right)\right]:\left[A p^{-1} c /\left(\rho_{P S} 4 \pi R^{3} / 3\right)\right]=3700: 1$. The adsorbed particles thus strongly dominate the measured signal.

As a further characteristic, the correlation functions in Figs. 4 and 5 contain a slow mode with a relaxation time $\tau_{2}$ between 7 and $42 \mathrm{~s}$. No systematic dependence of $\tau_{2}$ on $q_{\|}$or $q_{\perp}$ could be identified. On the one hand, the slow mode is outside of the experimental window where reliable data are expected. The TS140 vibration isolation table used in the experiment (see Sec. III) provides active damping in the frequency range $0.7 \mathrm{~Hz}-1 \mathrm{kHz}$ [26]. For lower frequencies comparable to $\tau_{2}^{-1}$, there is no isolation from external perturbations. On the other hand, the slow mode fits nicely to an exponential relaxation, which is not expected to be the case for external perturbations. Further, true external perturbations do not obey Gaussian statistics for the scattered field amplitude [27], and therefore they should increase the intercept of the correlation function to a value above 1 , which is not observed in the experiment. Finally, in actual measurements on different particles, we find a full relaxation of $g_{1}(t)$ within the reliable experimental window and a slow mode is absent. If the slow mode observed here would be caused by external perturbations, it should be present in other measurements as well. It thus appears that the water-oil interface is self-damping and therefore quite well behaved. The trick of pouring oil on water in order to tame the waves was known since ancient times. Here we find its application on a microscopic level.
A striking feature of the $g_{1}(t)$ data in Figs. 4 and 5 is the constant height of the plateau between the fast and the slow mode. There is a constant intensity ratio of the fast and the slow relaxation, independent of $q_{\|}$. This observation indicates that both modes are caused by the very same scattering units. Any interpretation where the two modes are assigned to the diffusion constants of two species of different size can be excluded, because the resulting different $q_{\|}$dependencies of the scattering intensities would result in a variation of the plateau height. The presence of a plateau indicates that the fast in-plane diffusion does not completely relax a fluctuation in the local interface coverage.

For an interpretation, we assume that electrostatic repulsion between the particles leads to the formation of a 2D hexagonal lattice at the interface, as observed by Pieranski for larger charged colloids [28]. Although for the investigated $c$ the zeta potential $\zeta=-14 \mathrm{mV}$ amounts to only $20 \%$ of the value observed at higher $c$ owing to desorption of PFO (see Sec. IV A), the remaining charge appears sufficient to provide significant repulsion. The diffusion in such a lattice consists of a short-range motion of a colloid around the equilibrium position ("rattling in a cage") without complete loss of coherence and longer range steps to the next equilibrium position ("cage release") [3,29]. In principle, the observed two-step relaxation of $g_{1}(t)$ fits into this picture. However, within the experimental errors the magnitude of $\tau_{2}$ compares well with $\tau_{0}$. The slow mode thus reflects rather the particle desorption, while a cage release within the plane, which might be significantly slower, is not resolved. Still, the presence of the interface lattice is crucial for understanding why $g_{1}(t)$ does not decay completely by the fast in-plane diffusion.

As a cross check of the interfacial structure, Fig. 6 shows the change of the scattering intensity for a scan of $q_{\|}$with $q_{\perp}$ and $p$ fixed. A broad peak confirms the presence of an interface lattice

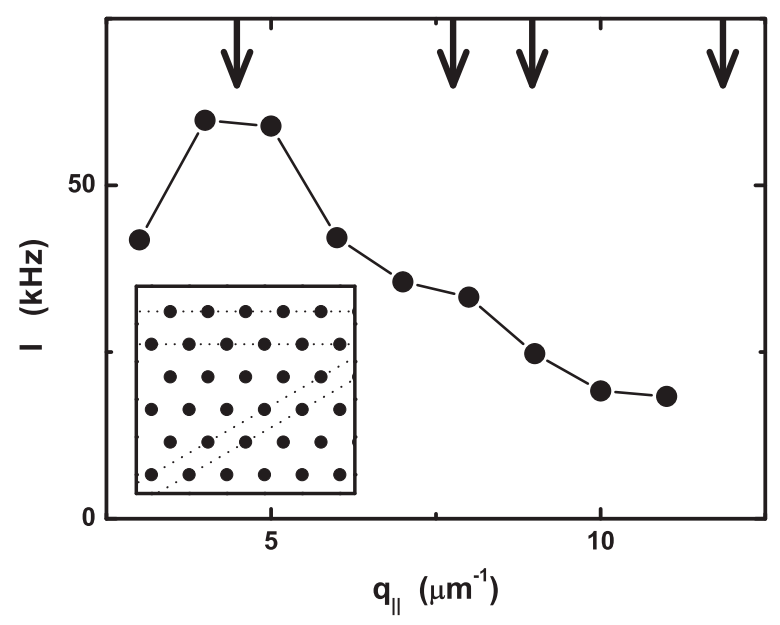

FIG. 6. Scattering intensity for EUR62 colloids at the heptanewater interface for $q_{\perp}=2 \mu \mathrm{m}^{-1}, p^{-1}=2 \mu \mathrm{m}$, and varying $q_{\|}$. The arrows indicate the expected peak positions of a hexagonal lattice with an interface coverage as detected by ellipsometry. The sketch in the inset shows a hexagonal lattice and two pairs of lattice lines that correspond to the first-order (upper line pair) and second-order (lower line pair) scattering peak. 
that is highly distorted. A more advanced data evaluation of such an interface static light-scattering measurement would require the knowledge of polarization- and geometry-dependent correction factors, as well as a description of the interface particle form factor. Because these factors have a rather smooth variation on the scattering angles, the location and the width of the peak are hardly affected, and an estimation from the raw data gives reliable results. For comparison, expected peak locations are calculated based on the interface coverage determined by ellipsometry (see Sec. IV B). The equilibrium distance $d$ between colloids is estimated from an equilateral triangular lattice element, where three colloids at the corners contribute $\frac{1}{6}$ of their interface coverage to the triangle area. The covered area fraction equals $\Phi$, and $d$ results as $d=R \sqrt{2 \pi /(\Phi \sqrt{3})}=1.6 \mu \mathrm{m}$. Scattering peaks expected at the locations $q_{\|, m, n}=4 \pi / d \sqrt{\left(m^{2}+n^{2}-m n\right) / 3}(m, n \in \mathbb{Z})$ are indicated by vertical arrows in Fig. 6 . The sketch in the inset of Fig. 6 indicates the lattice planes of the first- and secondorder scattering peaks (e.g., $q_{\|, 1,0}$ and $q_{\|, 1,2}$, respectively), while the third order (e.g., $q_{\|, 2,0}$ ) is located at twice the $q$ value of the first order. The prediction of the first-order peak matches nicely the experimental result and thus confirms the interface coverage determined by ellipsometry. A slightly increased scattering intensity close to the expected secondand third-order peak is consistent with the assumed model. Its weak magnitude and substantial width of the first-order peak indicate a high degree of disorder of the interface lattice.

It is instructive to compare the average distance a particle diffuses while it is absorbed with the average particle distance. During the average adsorption duration $\tau_{0}$, the root-meansquared diffusion distance $\sqrt{D_{\|} \tau_{0}}=0.94 \mu \mathrm{m}$ is roughly half the value of the lattice spacing. This estimation confirms that the slow mode is connected to desorption, not to the cage release. The interface structure of EUR62 is thus very soft and fluctuating. No slowing down of the diffusive relaxation at the peak position (de Gennes narrowing) is resolved. For bulk dynamics, such a $q$-dependent slowing down of the apparent diffusion constant is well established for hard spheres [29], while for soft spheres it is absent [3]. Note that such a slowing down is expected for a slow mode ("cage release"), not for the fast mode ("rattling").

A quantitative comparison of the mobility of particles trapped at the interface with their bulk diffusion gives further information about the interfacial state. For Eur62, the observed in-plane diffusion $\left(D_{\|}=0.44 \mu \mathrm{m}^{2} / \mathrm{s}\right)$ is slower by a factor 6 or 13 compared to the calculated colloidal diffusion in water $\left(D=2.5 \mu \mathrm{m}^{2} / \mathrm{s}\right)$ or in heptane $\left(D=6.1 \mu \mathrm{m}^{2} / \mathrm{s}\right)$, respectively. Note that the interface lattice cannot account for such a slowing down of the fast "cage rattling". The repulsive interactions between the particles enhance the restoring force for fluctuations, and thus the dynamic is rather speeded up within the lattice. For a solid-liquid interface, a weaker slowing down of the particle diffusion close to the interface observed by EWDLS was explained as an effect of hydrodynamic interaction between the particles and the immobile wall $[7,8]$. The average distance to the wall for particles was larger for this case compared to our measurements, where the colloids are adsorbed to the interface. A hydrodynamic interaction similar to the hard-wall case acts over a shorter distance and therefore can have a significant contribution to the friction, although the wall is not solid here and thus there is also a drag across the interface. Further friction could arise from the creation of capillary waves in particle motion and a possible interface distortion caused by the presence of the particle. For a movement of a particle, also the distortion has to be dragged along. The energy dissipated in the induced flow contributes to the friction. An investigation of the effect of particle size and shape on the interfacial friction is the subject of future work.

EWDLS measurements for "Harry" colloids at the dodecane-water interface are shown in Fig. 7. Although the data quality is lower than before, it is interesting to compare the dynamics of the two systems. Again a two-step decay is observed, which was transformed into a relaxation time distribution function $p(\tau)$ via the CONTIN software [30]. A fit of $p(\tau)$ with the sum of two log-normal distributions yields the average relaxation times $\bar{\tau}_{i}$ and the distribution widths $\sigma_{i}(i \in$ $\{1,2\})$, which in turn can be transformed to the initial slopes $\tau_{i}^{-1}=\bar{\tau}_{i}^{-1} \exp \left(\sigma_{i}^{2} / 2\right)$ of the two modes [31]. The inset of Fig. 7 shows a linear increase of $\tau_{1}^{-1}$ with $q_{\|}^{2}$, indicative for in-plane diffusion. The slope reads $D_{\|}=0.14 \mu \mathrm{m}^{2} \mathrm{~s}^{-1}$. A rough estimate of the position of the scattering peak is derived from EWDLS results, based on the assumption that the crossing point $q_{\|,+}$of the extrapolated $q_{\|}^{2}$-dependent diffusive fast dynamics and the extrapolated slow $\tau_{2}$ relaxation independent of $q_{\|}$cross at the peak position, i.e., $D_{\|} q_{\|,+}^{2}=\tau_{2}^{-1}$. The $q_{\|}$-independent relaxation time $\tau_{2}=2 \mathrm{~s}$ of the slow mode leads to $q_{\|,+}^{-1}=0.53 \mu \mathrm{m}$, in good agreement with $d=0.45 \mu \mathrm{m}$ calculated from the ellipsometry result for $\Phi$. Also for the "Harry" colloids, the picture of a diffusion in a 2D lattice and presumably slow desorption yields a consistent interpretation.

As compared to the calculated bulk diffusion for particles of similar radius in water $\left(D=4.1 \mu \mathrm{m}^{2} / \mathrm{s}\right)$ or dodecane ( $D=2.8 \mu \mathrm{m}^{2} / \mathrm{s}$ ), the interface diffusion is slowed down by a

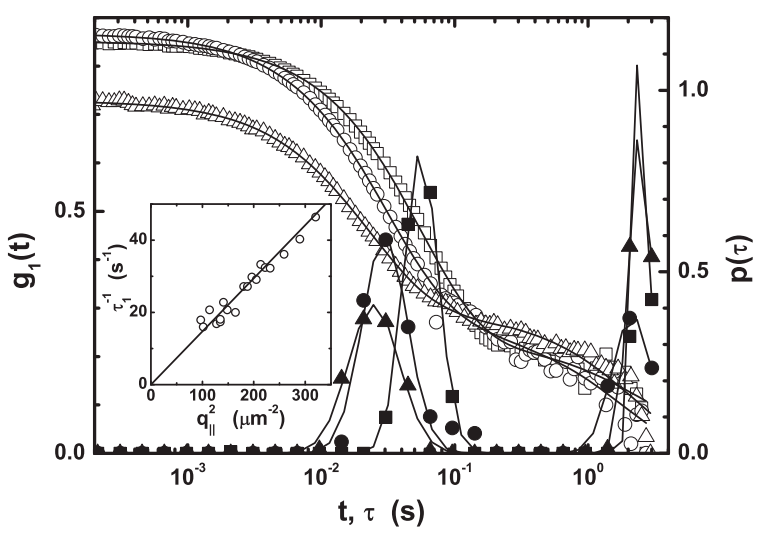

FIG. 7. Field correlation functions $g_{1}(t)$ and corresponding relaxation time distribution function $P(\tau)$ detected in total internal reflection geometry at the dodecane-water interface with adsorbed "Harry" particles for three different values of the parallel scattering vector component $q_{\|}\left(\square 9.8 \mu \mathrm{m}^{-1}\right.$, ○ $\left.14.6 \mu \mathrm{m}^{-1}, \triangle 17 \mu \mathrm{m}^{-1}\right)$ The penetration depth $p^{-1}=0.3 \mu \mathrm{m}$ of the evanescent wave and the perpendicular scattering vector component $q_{\perp}=10 \mu \mathrm{m}^{-1}$ are identical for the three measurements. Inset: Inverse relaxation time $\bar{\tau}^{-1}$ vs $q_{\|}^{2}$ with $p^{-1}$ and $q_{\perp}$ as before. 
factor 30 or 20, respectively. The comparison of the slowdown by a factor 6 for the Eur62 colloids and by a factor of 30 for the "Harry" colloids indicates a significant increase in friction for the smaller "Harry" colloids. Related investigations in the literature agree with an increased slowing down for smaller particles. Based on videomicroscopy on fluorescent rough silica particles of $R \approx 770 \mathrm{~nm}$ at an octanol-water interface, Vignati, Piazza, and Lockhart report no slowing down compared to bulk diffusion at low interface coverage and only a factor of 3 slowing down for an interfacial coverage as high as $50 \%$ [32]. Contrary findings of a slowing down by four orders of magnitude compared to the bulk diffusion of 4.6-nm-sized coated CdSe particles at a toluene-water interface determined by fluorescence photobleaching methods are reported by Lin et al. [33].

\section{CONCLUSIONS AND OUTLOOK}

In EWDLS measurements on charge-stabilized colloids adsorbed to an oil-water interface, a two-step decay of the correlation function was observed by EWDLS for two samples. The fast dynamic is independent of the scattering vector component $q_{\perp}$ perpendicular to the interface and depends only on the in-plane component $q_{\|}$. A broad peak in static interface light-scattering data for EUR62 hints at an interfacial lattice with high disorder, formed owing to electrostatic repulsion of the colloids. The discussion as a fast colloid diffusion around the equilibrium position and a slower desorption process leads to a consistent interpretation. A significant slowdown of the in-plane diffusion compared to the bulk diffusion was observed. These measurements demonstrate the possibilities of a newly built-up scattering goniometer for EWDLS at a liquid-fluid interface, which allows an independent variation of $q_{\|}, q_{\perp}$, and the penetration depth of the evanescent wave. The samples under study were originally considered to be the most simple test systems. However, these simple systems already demonstrate the complexity of the oil-water interface. More detailed investigations on the effects of particle size, shape, and concentration as well as the ionic strength in the aqueous phase on the interfacial particle concentration, location, and diffusion will form the subjects of future work.

\section{ACKNOWLEDGMENTS}

We thank Hubert Motschmann for initial help in setting up the Multiskop, and we thank Reinhard Miller for advice. Klaus Tauer is acknowledged for providing the samples. T.M. and R.S. thank Peter Schurtenberger and the Network of Excellence Softcomp for support. Financial support by the Max Planck Society and the Adolphe Merkle Foundation is gratefully acknowledged.

\section{APPENDIX A: COORDINATE TRANSFORMATIONS}

The tilting of the goniometer is shown in Fig. 8. With known lengths $L_{1}$ and $L_{2}$ of the lever arms and height difference $H$, the position $D$ of the linear stage (isel) can be transformed into a tilt angle $\chi$. The lengthy formula is omitted here. A general coordinate transformation based on rotation matrices is discussed by Germer and Asmail [34]. A simpler description

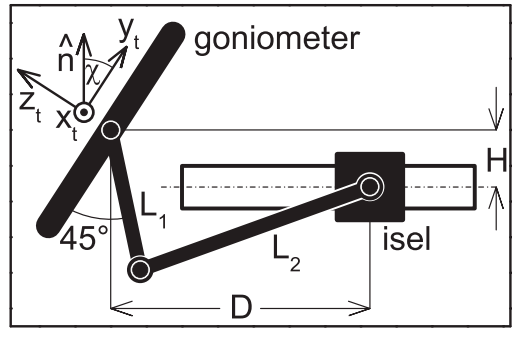

FIG. 8. Tilting mechanism of the goniometer by the isel linear stage via two lever arms of length $L_{1}$ and $L_{2}$. The $z$ axis of the coordinates is tilted by the angle $\chi$ relative to the surface normal $\hat{n}$ of a liquid interface.

for our scattering geometry is based on a coordinate system fixed to the goniometer, where the goniometer arms move within the $(x, y)$ plane and the axis for the tilting coincides with the $x$ axis (see Fig. 8). Unit vectors $\hat{l}$ and $\hat{d}$ along the laser arm and the detector arm, respectively, are

$$
\begin{gathered}
\hat{l}=\left(\sin \varphi_{t}, \cos \varphi_{t}, 0\right), \\
\hat{d}=\left(-\sin \psi_{t}, \cos \psi_{t}, 0\right) .
\end{gathered}
$$

Here, $\varphi_{t}$ and $\psi_{t}$ denote the angles of laser and detector arm in the goniometer plane, respectively. The negative sign in Eq. (A2), which implies an opposite orientation, is introduced for a simple version of the reflection law $\varphi_{t}=\psi_{t}(\chi=0)$ without a further sign included. The normal unit vector at the liquid interface reads

$$
\hat{n}=(0, \cos \chi, \sin \chi) .
$$

For polar coordinates, $\hat{n}$ marks the axis of symmetry. The latitudes $\varphi_{p}$ and $\psi_{p}$ of $\hat{l}$ and $\hat{d}$ become

$$
\begin{aligned}
\cos \varphi_{p} & =\hat{n} \cdot \hat{l}=\cos \chi \cos \varphi_{t}, \\
\cos \psi_{p} & =\hat{n} \cdot \hat{d}=\cos \chi \cos \psi_{t} .
\end{aligned}
$$

The in-plane geometry is calculated favorably with the projection operator $[\underline{1}-\hat{n}: \hat{n}]$, where 1 is the unit tensor and . : . marks the tensor product. The in-plane scattering vector component $q_{\|}$results as a projection of the difference of wave vectors for incident and detected light to the interface. With Eqs. (A1)-(A3), its magnitude reads

$$
q_{\|}=n_{1} k_{0}\left[\left(\sin \varphi_{t}-\sin \psi_{t}\right)^{2}+\left(\cos \varphi_{t}+\cos \psi_{t}\right)^{2} \sin ^{2} \chi\right]^{1 / 2} \text {. }
$$

The scalar product of the projected and then normalized wavevector components of incident and detected light yields $\cos \vartheta$, where $\vartheta$ is the in-plane scattering angle. The squared normal component $k_{i \perp}^{\prime 2}$ of the wave vector of the illuminating light in medium 2 results as a difference of the squared wave-vector modulus in medium 2 and the squared parallel component of the incident light. With Snell's law [Eq. (1)], the result reads

$$
k_{i \perp}^{\prime 2}=n_{1}^{2} k_{0}^{2}\left(\cos ^{2} \chi \cos ^{2} \varphi_{t}-\cos ^{2} \varphi_{0}\right) .
$$

An evanescent wave results for $k_{i \perp}^{\prime 2}<0$, so $p=\left|k_{i \perp}^{\prime}\right|$. A similar consideration yields the squared wave vector component $k_{s \perp}^{\prime}$ for the scattered light

$$
k_{s \perp}^{\prime 2}=n_{1}^{2} k_{0}^{2}\left(\cos ^{2} \chi \cos ^{2} \psi_{t}-\cos ^{2} \varphi_{0}\right) .
$$


For $k_{i \perp}^{\prime 2}<0$ and $k_{s \perp}^{\prime 2}>0, q_{\perp}=k_{s \perp}^{\prime}$. A special case occurs for $k_{s \perp}^{\prime 2}<0$. Owing to refraction at the interface, no scattered light that has been a plane wave in medium 2 can enter this regime. However, a field distribution of the scattered light similar to an evanescent wave is expected to show up at such a detection angle. It might be of high interest for samples where localized interface fluctuations are present, as, e.g., for the liquid crystals investigated previously [12].

For an inversion of formulas (A6)-(A8) for a standard EWDLS experiment $\left(\varphi_{p}>\varphi_{0}, \psi_{p}<\varphi_{0}\right)$, in a first step the in- plane vector components $k_{i \|}^{2}=n_{2}^{2} k_{0}^{2}+p^{2}$ and $k_{s \|}^{2}=n_{2}^{2} k_{0}^{2}-q_{\perp}^{2}$ for incident and scattered light are determined from $q_{\perp}$ and $p$. The normal components of wave vectors in medium 1 result as $k_{i \perp}^{2}=n_{1}^{2} k_{0}^{2}-k_{i \|}^{2}$ and $k_{s \perp}^{2}=n_{1}^{2} k_{0}^{2}-k_{s \|}^{2}$. Inserting $k_{i \|}, k_{s \|}$, and $q_{\|}$to the cosine rule yields $\vartheta$, so the geometry of the wave vectors $\mathbf{k}_{i}$ and $\mathbf{k}_{s}$ is known. The two vectors span the scattering plane. From geometric considerations the component of the normal unit vector $\mathbf{k}_{i} \times \mathbf{k}_{s} /\left|\mathbf{k}_{i} \times \mathbf{k}_{s}\right|$ perpendicular to the liquid interface equals $\sin \chi$. As a result,

$$
\sin ^{2} \chi=\frac{k_{i \|}^{2} k_{s \|}^{2}-\frac{1}{4}\left(k_{i \|}^{2}+k_{s \|}^{2}-q_{\|}^{2}\right)^{2}}{k_{i \|}^{2} k_{s \|}^{2}-\frac{1}{4}\left(k_{i \|}^{2}+k_{s \|}^{2}-q_{\|}^{2}\right)^{2}+k_{i \|}^{2} k_{s \perp}^{2}+k_{i \perp}^{2} k_{s \|}^{2}+\left(k_{i \|}^{2}+k_{s \|}^{2}-q_{\|}^{2}\right) k_{i \perp} k_{s \perp}} .
$$

With this input, $\varphi_{t}$ and $\psi_{t}$ are calculated from $\sin \varphi_{p}=$ $k_{i \|} /\left(n_{2} k_{0}\right), \sin \psi_{p}=k_{s \|} /\left(n_{2} k_{0}\right)$, and Eqs. (A4) and (A5).

The tilting of the goniometer affects also the polarization optics. A unit vector $\hat{s}$ indicating the $s$ polarization for the detector arm, e.g., reads $\hat{s}=\hat{n} \times \hat{d} /|\hat{n} \times \hat{d}|$. This expression is equal to a unit vector perpendicular to $\hat{d}$, which is rotated around $\hat{d}$ by a suitable angle. The comparison yields a shift of the analyzer angle scale,

$$
A_{p}=A-\arctan \frac{\tan \chi}{\sin \psi_{t}} .
$$

Here, $A_{p}$ is the analyzer angle in polar coordinates, while $A$ is the angle related to the goniometer coordinates. A similar calculation for the laser arm yields the azimuthal angle shifts of polarizer and compensator in polar coordinates.

\section{APPENDIX B: ADJUSTMENT OF THE APPARATUS}

The reliability of the results depends strongly on the alignment of the apparatus. Procedures known for the adjustment of light-scattering goniometers are applied to achieve such an adjustment.

The reference position of the laser arm for normal incidence is determined through the position of the beam reflected at the top interface of water in a vessel on the laboratory floor. The next step is to guide the incident light through the center of rotation of the goniometer. A fine needle is fixed in the center of the apparatus and the diffraction pattern of the laser light is observed at the opposite position, preferably magnified by a lens. The alignment of the needle into the central part of the beam yields a first reading $x_{1}$ on a micrometer screw. The laser arm is then moved by $180^{\circ}$ to the opposite position. The realignment of the needle to the center of the beam gives the second reading $x_{2}$. The needle is then moved to the central position $\left(x_{1}+x_{2}\right) / 2$ and the mirrors are adjusted to guide the beam to the position of the needle with a symmetric diffraction pattern. The laser beam now hits the center of rotation symmetrically. The needle can be used further to adjust a cylindrical lens on the laser arm, which focuses the beam on the needle (again symmetric pattern afterwards).

Once the beam is aligned, the cell must be positioned for its center to overlap the center of rotation. For horizontal alignment of the cell position (vertical beam), the partly filled cell acts as a cylindrical lens. A helpful tool is the quadrant diode, which allows a location of a beam impinging on the detector. The positions of the beam on the quadrant diode without the cell and with the partly filled cell have to coincide. For this step, another cylindrical lens on the detector arm is required to collimate the beam focused from the first lens on the laser arm. The alignment of this lens and the reference position of the detector arm for a central spot on the quadrant diode are correlated. The correct choice of the reference position of the detector arm is thus sufficient. It is determined by the condition that in opposite positions of laser and detector arm the laser should hit the quadrant diode centrally. In the correct distance from the center of rotation, the two lenses on the laser and detector arm compensate the lens effect of the completely filled cell. The vertical alignment of the cell position (horizontal beam) is now achieved as before for the horizontal alignment: The beam positions on the quadrant diode with and without the completely filled sample cell have to coincide. Optionally, two additional cylindrical lenses are introduced in the laser and detector arm for the focusing and recollimation in the second direction parallel to the axis of rotation. The optical fiber for light-scattering detection is adjusted to receive the laser beam in a position where the laser and detector arm are opposite without a sample cell. Because the laser beam passes through the center of rotation, the fiber also "sees" this center.

The interface level in a sample cell filled halfway by a liquid is checked in reflection geometry. The position of the laser arm defines the angle of incidence and the detector arm is set to the expected reflection. The filling level is adjusted until the reflected beam hits the quadrant diode in its center. An improvement is achieved when the centered position of the beam on the diode is checked for several angles of incidence, which span the widest possible range. Here, we implemented an automated search procedure scanning the position of the detector arm in order to locate the correct position where the beam is centered on the diode. The adjustment of the cell parallel to the axis of rotation has to result in a position where the interface remains in the scattering volume (defined by the incident laser beam and the ray accepted by the optical fiber) even when the goniometer is tilted. 
[1] B. J. Berne and R. Pecora, Dynamic Light Scattering (Dover, Mineola, NY, 2000).

[2] Dynamic Light Scattering, edited by W. Brown (Clarendon, Oxford, 1993).

[3] R. Sigel, S. Pispas, D. Vlassopoulos, N. Hadjichristidis, and G. Fytas, Phys. Rev. Lett. 83, 4666 (1999).

[4] R. Sigel, S. Pispas, N. Hadjichristidis, D. Vlassopoulos, and G. Fytas, Macromolecules 32, 8447 (1999); P. Holmqvist, S. Pispas, R. Sigel, N. Hadjichristidis, G. Fytas, and D. Vlassopoulos, ibid. 35, 3157 (2002); P. Holmqvist, S. Pispas, N. Hadjichristidis, G. Fytas, and R. Sigel, ibid. 36, 830 (2003).

[5] K. H. Lan, N. Ostrowsky, and D. Sornette, Phys. Rev. Lett. 57, 17 (1986).

[6] A. H. Marcus, B. H. Lin, and S. A. Rice, Phys. Rev. E 53, 1765 (1996).

[7] M. Hosoda, K. Sakai, and K. Takagi, Phys. Rev. E 58, 6275 (1998).

[8] P. Holmqvist, J. K. G. Dhont, and P. R. Lang, Phys. Rev. E 74, 021402 (2006); J. Chem. Phys. 126, 044707 (2007).

[9] E. Filippidi, V. Michailidou, B. Loppinet, J. Rühe, and G. Fytas, Langmuir 23, 5139 (2007).

[10] G. Fytas, S. H. Anastasiadis, R. Seghrouchni, D. Vlassopoulos, J. B. Li, B. J. Factor, W. Theobald, and C. Toprakcioglu, Science 274, 2041 (1996); B. Loppinet, G. Petekidis, G. Fytas, R. Rulkens, and G. Wegner, Langmuir 14, 4958 (1998); G. E. Yakubov, B. Loppinet, H. Zhang, J. Rühe, R. Sigel, and G. Fytas, Phys. Rev. Lett. 92, 115501 (2004); V. N. Michailidou, B. Loppinet, D. C. Vo, O. Prucker, J. Ruehe, and G. Fytas, J. Polym. Sci. B 24, 3590 (2006).

[11] C. S. Park, M. Copic, R. Mahmood, and N. A. Clark, Liq. Cryst. 16, 135 (1994).

[12] R. Sigel and G. Strobl, J. Chem. Phys. 112, 1029 (2000); Prog. Colloid Polym. Sci. 104, 187 (1997).

[13] B. H. Lin, S. A. Rice, and D. A. Weitz, J. Chem. Phys. 99, 8308 (1993); Phys. Rev. E 51, 423 (1995).

[14] R. Sigel, Curr. Opin. Colloid Interface Sci. 14, 426 (2009).
[15] Light Scattering by Liquid Surfaces and Complementary Techniques, edited by D. Langevin (Dekker, New York, 1992).

[16] J. Lekner, Theory of Reflection (Nijhof, Dordrecht, 1987).

[17] H. Harke, R. Teppner, O. Schulz, H. Orendi, and H. Motschmann, Rev. Sci. Instrum. 68, 3130 (1997).

[18] A. Erbe, K. Tauer, and R. Sigel, Langmuir 25, 2703 (2009).

[19] R. M. A. Azzam and N. M. Bashara, Ellipsometry and Polarized Light (Elsevier, Amsterdam, 1977).

[20] J. W. Benjamins, B. Joensson, K. Thuresson, and T. Nylander, Langmuir 18, 6437 (2002).

[21] J. Ricka, Appl. Opt. 32, 2860 (1993).

[22] A. Erbe, K. Tauer, and R. Sigel, Phys. Rev. E 73, 031406 (2006); Langmuir 23, 452 (2007); A. Erbe and R. Sigel, Eur. Phys. J. E 22, 303 (2007).

[23] A. Stocco and K. Tauer, Eur. Phys. J. E 30, 431 (2009).

[24] A. Stocco, K. Tauer, S. Pispas, and R. Sigel, Eur. Phys. J. E 29, 95 (2009).

[25] D. Schrader, in Polymer Handbook, edited by J. Brandrup, E. H. Immergut, and E. A. Grulke, 4th ed. (Wiley, New York, 1999).

[26] Instruction Manual for the TS-140 Active Vibration Isolation System (HWL Scientific Instruments GmbH).

[27] K. Schätzel, in Dynamic Light Scattering, edited by W. Brown (Clarendon, Oxford, 1993).

[28] P. Pieranski, Phys. Rev. Lett. 45, 569 (1980).

[29] P. N. Segre, S. P. Meeker, P. N. Pusey, and W. C. K. Poon, Phys. Rev. Lett. 75, 958 (1995); P. N. Segre and P. N. Pusey, ibid. 77, 771 (1996).

[30] S. W. Provencher, Comput. Phys. Commun. 27, 213 (1982).

[31] Z. Hordyjewicz-Baran, L. You, B. Smarsly, R. Sigel, and H. Schlaad, Macromolecules 40, 3901 (2007).

[32] E. Vignati, R. Piazza, and T. Lockhart, Langmuir 19, 6650 (2003).

[33] Y. Lin, A. Boeker, H. Skaff, D. Cookson, A. D. Dinsmore, T. Emrick, and T. P. Russell, Langmuir 21, 191 (2005).

[34] Th. A. Germer and C. C. Asmail, Rev. Sci. Instrum. 70, 3688 (1999). 